\title{
The SO(H)L(H) "O" drivers of oocyte growth and survival but not meiosis I
}

\section{T. Rajendra Kumar}

Department of Obstetrics and Gynecology, Division of Reproductive Sciences, Division of Reproductive Endocrinology, Charles Gates Stem Cell Center, University of Colorado Anschutz Medical Campus, Aurora, Colorado, USA.

\begin{abstract}
The spermatogenesis/oogenesis helix-loop-helix (SOHLH) proteins SOHLH1 and SOHLH2 play important roles in male and female reproduction. Although previous studies indicate that these transcriptional regulators are expressed in and have in vivo roles in postnatal ovaries, their expression and function in the embryonic ovary remain largely unknown. Because oocyte differentiation is tightly coupled with the onset of meiosis, it is of significant interest to determine how early oocyte transcription factors regulate these two processes. In this issue of the $\mathrm{JCl}$, Shin and colleagues report that SOHLH1 and SOHLH2 demonstrate distinct expression patterns in the embryonic ovary and interact with each other and other oocyte-specific transcription factors to regulate oocyte differentiation. Interestingly, even though there is a rapid loss of oocytes postnatally in ovaries with combined loss of Sohlh1 and Sohlh2, meiosis is not affected and proceeds normally.
\end{abstract}

\section{Introduction}

In the XX mouse embryo, the primordial germ cells give rise to oogonia, the oocyte precursors (1-4). In the embryonic female mouse gonad, germ cells are enclosed in germline cysts and enter meiosis. After birth, germline cyst breakdown occurs, and primordial follicles are assembled when oocytes become surrounded by the somatic pregranulosa cells $(1,3,5,6)$. Female germ cells are developmentally arrested during late gestation at the diplotene stage of the prophase of the first meiotic division. This prophase I arrest is maintained until ovulation, when the luteinizing hormone surge stimulates meiotic resumption $(1,3$, $5,7)$. In coordination with the surrounding somatic (granulosa) cells, the oocyte must begin its journey forward by simultaneously undergoing growth (and survival) and also meiosis - two interrelated processes (1, 7-9). Several transcription factor-mediated networks orchestrate the early differentiation program of oogenesis/folliculogenesis $(2,4,8,10-12)$. Null mutations in the granulosa cell-specific master transcription regulator forkhead box L2 (Foxl2) result in an impaired somatic cell differentiation program, leading to problems in oocyte maintenance $(1,2,10,11,13)$. Similarly, mice lacking key oocyte transcription factors demonstrate disrupted postnatal ovarian development $(2,4,12,14)$. However, the in vivo roles of oocyte-specific factors that control meiosis and oocyte differentiation in the embryonic ovary are less understood. Spermatogenesisand oogenesis-specific basic helix-loophelix (SOHLH) transcription factors 1 and 2 (SOHLH1 and SOHLH2) are key players in early folliculogenesis $(2,14-17)$. These transcription factors are also expressed during early testis development in undifferentiated spermatogonia and are important regulators of spermatogenesis $(15,17)$. In this issue of the JCI, Shin et al. have used a variety of elegant mouse genetic tools to delineate the embryonic roles of SOHLH proteins in early oocyte differentiation and meiosis in vivo (18).

Related Article: p. 2106

Conflict of interest: The author has declared that no conflict of interest exists.

Reference information: / Clin Invest. 2017;127(6):2044-2047. https://doi.org/10.1172/JCI94665.

Compartmentalization of SOHLH1 and SOHLH2 proteins SOHLH1 and SOHLH2 proteins are encoded by distinct genes and consist of 357 and 467 amino acids, respectively. The Rajkovic laboratory previously generated specific and well-characterized antibodies against each of these two proteins $(15,17,19)$. However, the embryonic expression patterns of these proteins have not been tested before. Using these antibodies and gene expression data sets available from public resources, Shin et al. tested the expression of mRNA (by real-time PCR) and the corresponding proteins SOHLH1 and SOHLH2 (by immunofluorescence and Western blot analysis) in mouse embryos beginning at E11 up to P2 (18). They also evaluated the expression of several well-characterized oocyte transcription factors for comparison. Sohlh2 transcripts were detected as early as E12.5, before oocytes enter meiosis, and the onset of expression of Sohlh1. Another oocyte transcription factor, NOBOX oogenesis homeobox (Nobox), was not detected until E15.5. Western blot analysis revealed SOHLH2 expression at E12.5 and SOHLH1 expression at E16.5. Interestingly, immunolocalization experiments showed SOHLH2 protein to be confined to the cytoplasm prior to E15.5 and predominantly limited to the nucleus afterward, at which time SOHLH1 expression commenced and both proteins colocalized. This colocalization persisted even after birth, when both SOHLH1 and SOHLH2 were detected in oocytes of primordial and primary follicles.

Notably, loss of SOHLH1 in ovaries did not result in loss of SOHLH2, whereas loss of SOHLH2 in ovaries resulted in loss of SOHLH1. Moreover, expression of SOHLH2 was confined to the cytoplasm of Sohlh1-KO ovaries (18). Collectively, these studies indicated an epistatic genetic interaction between SOHLH2 and SOHLH1, with SOHLH2 hierarchically placed above SOHLH1. Furthermore, these studies 
A

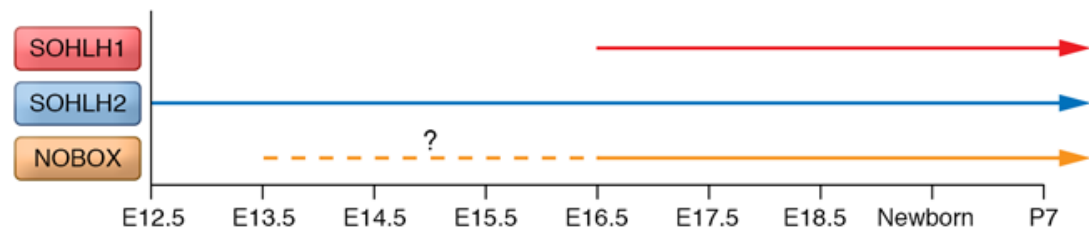

B

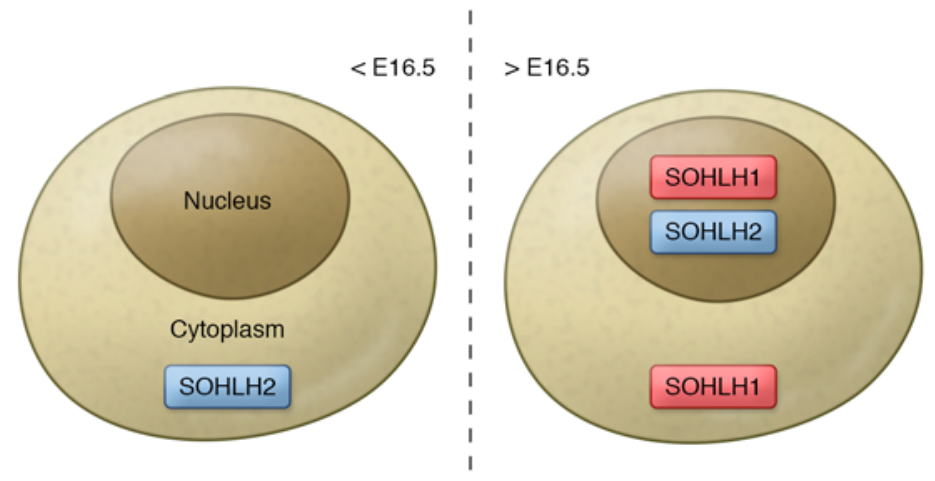

identified age-dependent spatio-temporal differences in the expression of SOHLH1 and SOHLH2, with SOHLH2 nuclear import dependent on the presence of SOHLH1. Whether the early onset and the cytoplasmic localization of SOHLH2 prior to SOHLH1 expression have any biological significance remains to be tested.

\section{SOHLH1 and SOHLH2 are critical regulators of oocyte growth and differentiation}

Previous studies have indicated in vivo genetic interactions between SOHLH1 and SOHLH2 and have characterized the ovarian phenotypes in mice lacking either Sohllh1 or Sohlh2 $(2,14,16)$. Using these available genetic tools, Shin and colleagues have now created mice lacking both Sohlh1 and Sohlh2, allowing the effects of combined deficiency of SOHLH1 and SOHLH2 on oogenesis to be tested (18). Surprisingly, double mutants were indistinguishable from single mutants their ovaries were hypoplastic, they displayed diminished early-stage follicles (primordial and primary) by weeks 1 and 2 , and they lost the majority of oocytes by the time they reached reproductive maturity (7 weeks). Because of the apparent epistatic interaction between SOHLH2 and SOHLH1, Shin et al. devised a set of clever genetic experiments to test whether the loss of SOHLH1 contributes to ovarian phenotypes in Sohlh2-KO mice. Specifically, the authors designed two independent floxed chloramiphenicol acetyl transferase (CAT) transgene (Sohlh1 or Sohlh2) mCher$r y$ reporter FLAG-tagged constructs driven by the chicken $\beta$-actin promoter. In the absence of CRE recombinase, the floxed $C A T$ gene prevents transgene expression; however, when expression of CRE is driven by oocyte-specific promoters, it allows expression of the Sohlh1 (known as the CCS1 transgene) or the Sohlh2 (known as the CCS2 transgene) transgene. Moreover, this system provides the ability to visualize the cells (via mCherry and the FLAG tag) to evaluate recombination efficiency.

In one set of experiments, DEAD (Asp-Glu-Ala-Asp) box polypeptide-Cre (Ddx4-Cre) was used, as $D d x 4$ expression is not suppressed by loss of either Sohlh1 or Sohlh2 (20). This allowed Sohlh1 expression from the CCS1 transgene in the Sohlh1null genetic background after the onset of meiosis, specifically between E15.5 and E18.5, when the $D d x 4$ promoter is activated. Moreover, $D d x 4$-driven expression of Sohlh1 rescued the ovarian and fertility phenotypes associated with SOHLH1 deficiency. Strikingly, SOHLH2, which remained cytoplasmic in SOHLH1-deficient oocytes, was shuttled to the nucleus in Solhlh1-null mice expressing the CCS1 transgene, further confirming that nuclear transport and compartmentalization of SOHLH2 is SOHLH1 dependent.

In a second set of experiments, Shin et al. showed that expression of the same SOHLH1-encoding CCS1 transgene in a
Figure 1. Expression of SOHLH1 and SOHLH2 in a mouse oocyte. The temporal expression (A) and spatial expression (shown in the cytoplasm or the nucleus in B) of SOHLH1 and SOHLH2 proteins are distinct at different embryonic time points during oocyte development in the mouse ovary. The expression of another oocyte transcription factor, NOBOX, closely overlaps with that of SOHLH1, although its weak expression is noted at early time points (indicated by a question mark above the dashed line in A). Shin et al. demonstrate that $\mathrm{SOHLH} 1$ is required for nuclear localization of SOHLH2 in germ cells in the mouse ovary after, but not before, E16.5 (B).

Sohlh2-null genetic background failed to rescue Sohlh2 deficiency (18). The transgene itself was not defective on the Sohlh2null background and was expressed, as evidenced by mCherry expression. Two possibilities exist for this lack of Sohlh2 rescue by the Sohlh1 transgene. First, proteins other than SOHLH1 may be needed for SOHLH2 actions, or, second, the formation of SOHLH2-SOHLH1 heterodimers is essential for full downstream effects of SOHLH2.

In a third set of experiments, the second transgene (CCS2, which expresses Sohlh2) was introduced onto the Sohlh2null background. Intriguingly, expression of the Sohlh2 transgene failed to rescue phenotypic defects or restore expression of SOHLH1 in Sohlh2-null mice (18). One potential reason for this result could be a lack of heterodimer formation between SOHLH1 and SOHLH2 proteins, as described above. However, these predictions must be experimentally tested in future studies.

In a final set of experiments, Shin and colleagues tested whether Sohlh1-null mice could be rescued by postnatal expression of CCS1 using oocyte-specific growth differentiation factor 9-Cre (Gdf9-Cre) (21). In this line, CRE expression is initiated at PD3 in primordial follicles and advanced-stage follicles. Sohlh 1 transgene activation postnatally by Gdf9-Cre only partially rescued Sohlh 1 deficiency (18). Although these rescue mice displayed a reduced primordial follicle pool 
in their ovaries, they were fertile when mated and successfully produced viable offspring. The residual SOHLH1:SOHLH2 heterodimers that persisted were perhaps functionally sufficient to result in the observed partial rescue of Sohlh1-null mice in this experiment. Together, these novel mouse genetic tools have proved invaluable in deciphering the embryonic roles of SOHLH1 and SOHLH2 proteins.

\section{SOHLH1 and SOHLH2 orchestrate oocyte-specific transcriptional programs}

In the mouse ovary, E15.5 appears to be a critical time point. At this time, SOHLH1 expression commences, and, concomitantly, SOHLH2 translocates to the nucleus, as demonstrated by Shin et al. in the current study (18). Most important, embryonic expression of many genes that encode oocyte transcription factors also commences at E15.5 and significantly overlaps Sohlh1 expression. Shin et al. used a panel of antibodies against SOHLH1, NOBOX, and LIM homeobox protein 8 (LHX8) proteins (whose RNA expression profiles closely overlap) and evaluated the expression of these proteins in embryonic ovaries. Expression of the transcription factors NOBOX and LHX8 was initiated in $\mathrm{SOHLH}^{+}$cells within E16.5 ovaries. One day later, by E17.5, the majority of oocytes (>90\%) were found to be SOHLH1 ${ }^{+}$ $\mathrm{LHX}^{+} \mathrm{NOBOX}^{+}$and were identified as a distinct subset of germ cells. Moreover, loss of SOHLH1, SOHLH2, or both reduced NOBOX and LHX8 expression in ovaries but did not affect individual intracellular localization. However, in nearly half of the oocytes, NOBOX and LHX8 did not colocalize in the absence of SOHLH1 and SOHLH2. Additionally, loss of LHX8 appeared to diminish expression of SOHLH1 in the embryonic ovaries of Lhx8-null mice. In Sohlh1 and Sohlh2 single-KOs, LHX8-dependent expression of KIT proto-oncogene receptor tyrosine kinase (Kit), a receptor for the steel ligand and important for germline differentiation, was downregulated. Thus, SOHLH1 and SOLHL2, together with LHX8 and other oocyte transcription factors, mutually regulate the appropriate expression of one another and orchestrate the transcriptional network of interactions in the embryonic gonad.

\section{Meiosis proceeds normally without SOHLH1 and SOHLH2}

Next, Shin et al. addressed how loss of SOHLH1 and SOHLH2 influences meiosis (18). Oocytes enter meiosis in the mouse embryonic ovary at approximately E13.5, proceed to the zygotene and pachytene stages by E16.5, and arrest in the diplotene stage before birth $(1,7)$. Rapid oocyte loss is a feature of mice lacking key meiosis-specific genes $(1,2,12,14)$ and is a common feature observed in ovaries of Sohlh1- and Sohlh2-KO mice. Particularly, in the absence of SOHLH1 and SOHLH2, oocytes are rapidly lost after birth, at a time when most oocytes have entered the diplotene stage of meiosis. To identify whether gross perturbations in meiosis could account for rapid oocyte loss in Sohlh1and Sohlh2-KO mouse ovaries, Shin et al. performed RNA-sequencing (RNA-seq) analysis on ovaries from newborn mice. Expression of many meiosis-specific genes and those that disrupt early oogenesis was not significantly different in the absence of SOHLH1 and SOHLH2, indicating that early oocyte growth and survival may be regulated by factors independent of meiosis. This observation is very significant, as SOHLH2 expression nearly coincides with the onset of meiosis. Shin and colleagues also generated an exhaustive list of genes that are misregulated in the ovaries of newborn Sohlh1 and Sohlh2 single- and double-KO mice. Among the downregulated genes were many histone-encoding transcripts of unknown significance and, as predicted, several oocyte-specific mRNAs. Likewise, several testis-specific transcripts were upregulated, reinforcing the prevailing idea that oocytes normally repress male germline differentiation in the ovary.

\section{Summary and future directions}

Shin et al. used a variety of mouse genetic tools and conducted a beautiful expression analysis to demonstrate the in vivo roles of SOHLH1 and SOHLH2, proteins whose expression overlaps in the embryonic ovary (Figure 1). These studies identified a subset of embryonic female germ cells with a unique expression signature of key transcription factors and also provided two major insights into early oocyte differentiation. First, functional heterodimers between SOHLH1 and SOHLH2 proteins must exist in vivo. Second, SOHLH1 and
SOHLH2 are not required for meiosis, and yet loss of these transcriptional regulators results in rapid oocyte loss after birth. The studies by Shin et al. strongly support the recent observations made in the Page laboratory (9). Several future studies could be worth performing to further gain insights into the roles of SOHLH1 and SOHLH2 in oocyte biology. First, the in vivo significance of cytosolic SOHLH2, if any, may be studied by deleting Sohlh2 as early as E12.5. Similarly, a Sohlh2 transgene could be expressed at this time point on a Sohlh2null background to determine whether Sohlh2 deficiency could be fully rescued. Second, with the availability of a number of mouse strains lacking early oocyte-specific factors, it would be feasible to generate double or triple mutants lacking, for example, combinations of Sohlh1/Nobox, Sohlh1/Lhx8, Sohl2/Nobox, Sohl2/Lhx8, Sohlh1/Sohlh2/Nobox, or Sohlh1/Sohlh2/ Lhx8. Analysis of these double and triple mutants may definitively establish the roles of these factors in meiosis. Third, in vivo studies by Shin et al. suggest the existence of potential functional heterodimers of SOHLH1 and SOHLH2. This could be tested by expressing a bicistronic transgene cassette encoding both SOHLH1 and SOHLH2 proteins in mice with combined Sohlh1 and Sohlh2 deficiency. Fourth, because the transgene-derived SOHLH1 and SOHLH2 proteins contain FLAG tags, direct immunoprecipitation of protein complexes in ovarian extracts, followed by mass spectrometric analysis, could be performed to identify interactions between SOHLH1, SOHLH2, and other potential protein partners in oocytes. Fifth, meiosis analysis was restricted to only gene expression studies; therefore, detailed studies examining synapse formation and progression could be performed by a variety of methods (22). Finally, RNA-seq data generated by Shin et al. could also be exploited for future functional studies, particularly, if downregulation of histone mRNAs could somehow be linked to oocyte loss early after birth in double and single Sohlh1- and Sohlh2-null mutants.

\section{Acknowledgments}

I thank Francesca Duncan of Northwestern University for a critical reading of the manuscript and helpful suggestions on the manuscript. Work done in the 
author's laboratory was supported by NIH grants AG029531 and HD081162, and the Makowski Endowment.

Address correspondence to: T. Rajendra Kumar, Edgar L and Patricia M Makowski Professor, Department of Obstetrics and Gynecology, University of Colorado Anschutz Medical Campus, 12700 E. 19th Ave, 3100B, RC-2 Bldg., Aurora, Colorado 80045, USA. Phone: 303.724.8689. E-mail:raj.kumar@ucdenver.edu.

1. Albertini DF. The mammalian oocyte. In: Plant T, Zeleznik A, eds. Knobil and Neill's Physiology of Reproduction. 4th ed. New York, New York, USA: Elsevier; 2014:59-97.

2. Jagarlamudi K, Rajkovic A. Oogenesis: transcriptional regulators and mouse models. $\mathrm{Mol} \mathrm{Cell}$ Endocrinol. 2012;356(1-2):31-39.

3. Pepling ME. From primordial germ cell to primordial follicle: mammalian female germ cell development. Genesis. 2006;44(12):622-632.

4. Rodrigues P, Limback D, McGinnis LK, Plancha CE, Albertini DF. Oogenesis: prospects and challenges for the future. J Cell Physiol. 2008;216(2):355-365.

5. Pepling ME. Follicular assembly: mechanisms of action. Reproduction. 2012;143(2):139-149.

6. Pepling ME, de Cuevas M, Spradling AC. Germline cysts: a conserved phase of germ cell development? Trends Cell Biol. 1999;9(7):257-262.

7. Cohen PE, Holloway JK. Mammalian meiosis. In: Plant T, Zeleznik A, eds. Knobil and Neill's Physiology of Reproduction. 4th ed. New York, New York, USA: Elsevier; 2014:5-57.

8. Albertini DF. The structural basis of oocyte-granulosa cell communication. Ernst Schering Res Found Workshop. 2002;(41):101-110.

9. Dokshin GA, Baltus AE, Eppig JJ, Page DC. Oocyte differentiation is genetically dissociable from meiosis in mice. Nat Genet. 2013;45(8):877-883.

10. Albertini DF, Barrett SL. Oocyte-somatic cell communication. Reprod Suppl. 2003;61:49-54.

11. Nicol B, Yao HH. Building an ovary: insights into establishment of somatic cell lineages in the mouse. Sex Dev. 2014;8(5):243-251.

12. Zheng P, Dean J. Oocyte-specific genes affect folliculogenesis, fertilization, and early development. Semin Reprod Med. 2007;25(4):243-251.

13. Baron D, et al. Foxl2 gene and the development of the ovary: a story about goat, mouse, fish and woman. Reprod Nutr Dev. 2005;45(3):377-382.

14. Pangas SA, et al. Oogenesis requires germ cell-specific transcriptional regulators Sohlh1 and Lhx8. Proc Natl Acad Sci U S A. 2006;103(21):8090-8095.

15. Ballow D, Meistrich ML, Matzuk M, Rajkovic A.
Sohlh1 is essential for spermatogonial differentiation. Dev Biol. 2006;294(1):161-167.

16. Choi Y, Yuan D, Rajkovic A. Germ cell-specific transcriptional regulator sohlh2 is essential for early mouse folliculogenesis and oocyte-specific gene expression. Biol Reprod. 2008;79(6):1176-1182.

17. Suzuki H, et al. SOHLH1 and SOHLH2 coordinate spermatogonial differentiation. Dev Biol. 2012;361(2):301-312.

18. Shin YH, et al. Transcription factors SOHLH1 and SOHLH2 coordinate oocyte differentiation without affecting meiosis I. J Clin Invest. 2017;127(6):2106-2117.

19. Ramaswamy S, et al. Spermatogonial SOHLH1 nucleocytoplasmic shuttling associates with initiation of spermatogenesis in the rhesus monkey (Macaca mulatta). Mol Hum Reprod. 2014;20(4):350-357.

20. Park ES, Tilly JL. Use of DEAD-box polypeptide-4 (Ddx4) gene promoter-driven fluorescent reporter mice to identify mitotically active germ cells in post-natal mouse ovaries. Mol Hum Reprod. 2015;21(1):58-65.

21. Lan ZJ, Xu X, Cooney AJ. Differential oocyte-specific expression of Cre recombinase activity in GDF-9-iCre, Zp3cre, and Msx2Cre transgenic mice. Biol Reprod. 2004;71(5):1469-1474.

22. Gray S, Cohen PE. Control of meiotic crossovers: from double-strand break formation to designation. Annu Rev Genet. 2016;50:175-210. 